\title{
Tentativas de suicídio em jovens: aspectos epidemiológicos dos casos atendidos no setor de urgências psiquiátricas de um hospital geral universitário entre 1988 e $2004^{1}$
}

\author{
Suicide attempts among adolescents: epidemiological \\ aspects of patients cared for in a university hospital \\ psychiatric emergency unit between 1988 and 2004
}

\author{
Ana Maria Fortaleza Teixeira FICHER ${ }^{2}$ \\ Gerson Antonio VANSAN ${ }^{3}$
}

\begin{abstract}
Resumo
Este estudo teve por objetivo analisar, comparativamente, a distribuição temporal de todos os casos de pacientes adolescentes, com idade entre 10 e 24 anos, atendidos no setor de urgências psiquiátricas de um grande hospital geral universitário, após tentativas de suicídio e uso/abuso de substâncias psicoativas, durante o período de 1988 a 2004. Estudou-se, especificamente, os casos de tentativas de suicídio, considerando sua distribuição quanto à idade, sexo e método utilizado. A partir de um estudo de casos, apontam-se dados sobre os motivos desencadeantes das tentativas e sobre as histórias de vida desses pacientes, possivelmente relacionados à ocorrência desse comportamento. Os dados foram obtidos por meio de um levantamento retrospectivo de todos os casos de pacientes adolescentes atendidos no setor de urgências psiquiátricas do Hospital das Clínicas da Faculdade de Medicina de Ribeirão Preto, da Universidade de São Paulo, no período de 1988 a 2004, após tentativas de suicídio e uso/abuso de substâncias psicoativas, e de dez histórias clínicas obtidas por meio de entrevistas realizadas com pacientes adolescentes, selecionados ao acaso, atendidos no setor por tentativas de suicídio. Os resultados mostraram uma forte correlação positiva entre os números de casos anualmente atendidos por tentativas de suicídio e os devidos ao uso/abuso de substâncias psicoativas, tanto para os adolescentes do sexo masculino ( $r=0,70, p=0,0037)$, quanto para os do sexo feminino ( $r=0,81$, $p=0,0002$ ). As tentativas de suicídio foram significativamente mais freqüentes no sexo feminino, e o método mais utilizado para esse comportamento foi o envenenamento, preferencialmente com medicamentos. As histórias clínicas mostraram que a maioria desses adolescentes era proveniente de lares desestruturados (broken-homes), predominantemente por separação dos pais, e que a tentativa ocorreu com mais freqüência após discussão com pessoas significativas do núcleo sócio-familiar. Sem ignorar os problemas metodológicos existentes, é razoável supor que os fatos observados neste trabalho refletem a ocorrência de suicídio e uso/abuso de substâncias psicoativas na população geral, ou indicam, ao menos, que eles evidenciam um sério problema médico-psicológico em nível assistencial entre os adolescentes e adultos jovens.
\end{abstract}

Unitermos: Adolescentes. Epidemiologia. Tentativas de suicídio.

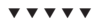

1 Artigo elaborado a partir da dissertação de A.M.F.T. FICHER, intitulada "Tentativas de suicídio de adolescentes atendidos no setor de urgências psiquiátricas de um hospital geral". Faculdade de Medicina de Ribeirão Preto, Universidade de São Paulo, 2000. Apoio financeiro: Coordenação de Aperfeiçoamento de Pessoal de Nível Superior.

2 Universidade de São Paulo, Faculdade de Medicina de Ribeirão Preto, Hospital das Clínicas, Unidade de Emergência. Ribeirão Preto, SP, Brasil.

3 Universidade de São Paulo, Faculdade de Medicina de Ribeirão Preto, Departamento de Neurologia, Psiquiatria e Psicologia Médica. R. Tenente Catão Roxo, 2650, Campus Universitário, Monte Alegre, 14051-140, Ribeirão Preto, SP, Brasil. Correspondência para/Correspondence to: G.A. VANSAN. E-mail: <gavansan@fmrp.usp.br>. 


\begin{abstract}
The objective of this study was to perform a comparative analysis of the temporal distribution of all cases involving adolescents between 10 and 24 years of age cared for in the psychiatric emergency unit of a large university hospital during the period from 1988 to 2004, as a result of attempted suicide or the use/abuse of psychoactive substances. Cases of attempted suicide, in particular, were studied by taking into consideration their distribution according to age, gender, and attempted method of suicide. By using case studies, data were collected in respect of the motives which triggered the suicide attempt as well as the life history of these patients which could possibly be related to the occurrence of this behavior. Data were obtained from a retrospective survey of all cases of adolescent patients who had attempted suicide and who had used/abused psychoactive substances and who attended the psychiatric emergency unit of the Clinical Hospital of the Faculty of Medicine at the University of São Paulo in Ribeirão Preto, between 1988 and 2004, and also from 10 clinical histories obtained by means of interviews with adolescent patients, selected at random, attending the unit as a result of attempted suicide. The results showed a strong positive correlation between the number of cases involving suicide attempts and the number of cases involving psychoactive substance use/ abuse for both male $(r=0.70 ; p=0.0037)$ and female adolescents $(r=0.81 ; p=0.002)$. Suicide attempts were found to be significantly more frequent among female adolescents, where the preferred method of suicide is poisoning, using prescription drugs. The clinical histories of the cases analyzed showed that the majority of these adolescents come from broken-homes, predominantly as a result of parental separation, and that the suicide attempts were often the result of arguments with close family members. Being aware of the methodological issues, it is reasonable to suppose that the facts observed in this study would be reflected in the general population or, at least, that they indicate a serious medical-psychological problem at the welfare level among adolescents and young adults.
\end{abstract}

Uniterms: Adolescents. Epidemiology. Suicide attempts.

Não somente em números absolutos, mas também em termos de sofrimento humano e em termos econômicos, o fenômeno do suicídio e a sua tentativa, em vários países do mundo, é um problema de saúde pública de primordial importância, particularmente entre adolescentes e adultos jovens (Diekstra, 1993).

Dados do setor de urgências psiquiátricas do Hospital das Clínicas da Faculdade de Medicina de Ribeirão Preto (HCFMRP-USP) revelaram que 23\% de todos os atendimentos ali realizados durante o período de 1988 a 1993 foram prestados a adolescentes. Destes, os devidos a tentativas de suicídio foram, em números, significativamente mais freqüentes nas mulheres (77\%) do que nos homens (23\%), sendo crescente durante o período (Teixeira \& Luis, 1997a).

Dados relativos ao período de 1988 a 1991 nesse setor revelaram uma proporção anual de atendimento a casos de tentativas de suicídio de adolescentes, em relação ao total anual das tentativas atendidas, variando de 40,0\% a 51,0\% durante o período (Teixeira \& Luis, 1997b). Ainda neste setor, Avanci, Pedrão e Costa Júnior (2005), examinando o perfil epidemiológico desses atendimentos em adolescentes, encontraram também uma proporção maior dos casos no sexo feminino (77,8\%), na faixa etária de 15 a 19 anos, sendo a ingestão de medicamentos o procedimento mais utilizado nas suas tentativas de suicídio.

Werneck, Hasselmann, Phebo, Vieira e Gomes 362 (2006), estudando também o perfil dos casos de tentativas de suicídio atendidos em um hospital geral do Rio de Janeiro, entre abril de 2001 e março de 2002, observaram que a maioria deles (68\%) era do sexo feminino, sendo quase dois terços das mulheres (62\%) e 42\% dos homens com menos de 25 anos de idade. Os procedimentos mais utilizados pelas mulheres foram, na mesma proporção, a ingestão de pesticidas e de medicamentos, e o mais empregado pelos homens, em dois terços dos casos, a ingestão de pesticidas.

Lamentavelmente, em nosso meio, a ausência de adequadas estatísticas sobre esse comportamento torna difícil o desenvolvimento periódico de estudos epidemiológicos analisando tendências evolutivas das taxas de tentativas de suicídio em comunidades diversas. Entretanto, para algumas comunidades, embora ainda escassos, há estudos indicando estimativas pontuais de taxas desse comportamento obtidas a partir de dados de registros hospitalares. Em Campinas (SP), por exemplo, Cassorla (1992), utilizando esses dados e visitando esses casos em seus domicílios, estimou uma taxa de 154 casos por 100 mil habitantes em 1980, dos quais 75\% haviam ocorrido em menores de 27 anos, e predominantemente no sexo feminino. Em Ribeirão Preto (SP), Andrade (1979), investigando todos os casos de tentativas de suicídio atendidos nos hospitais e prontos-socorros da cidade, no ano de 1977, observou a predominância desse comportamento no sexo feminino em relação ao masculino em uma proporção de $3: 1$, sendo que a maior taxa entre as mulheres 
(812,26/100 mil habitantes) foi encontrada em adolescentes na faixa etária de 15 a19 anos e, entre homens (281,03/100 mil habitantes), em jovens na faixa de 25 a 29 anos.

Assim, apesar das dificuldades metodológicas existentes na medição da verdadeira magnitude do fenômeno, esses resultados sugerem que as tentativas de suicídio, especialmente as que ocorrem entre adolescentes e adultos jovens, representam um importante problema de saúde pública, que demanda esforços contínuos de pesquisa, visando à identificação de fatores de risco que possam contribuir para o desenvolvimento de estratégias preventivas e assistenciais.

Nesse sentido, há estudos apontando fortes evidências de que o aumento da incidência do abuso/ dependência de substâncias psicoativas observado entre os adolescentes nas últimas décadas, em vários países, esteve significativamente associado ao crescimento das taxas de comportamentos suicidas (tentativas e suicídios completos) desses jovens, no mesmo período (Crumley, 1990).

No Brasil, dados apresentados por D'Oliveira (2000-2002) sobre 171 casos de tentativas de suicídio, sendo a maioria dos homens (79\%) e das mulheres (71\%) com idades entre 15 e 44 anos, apontam também fortes evidências sobre essa associação em nosso meio, indicando que 50\% dos homens e 15\% das mulheres que tentaram o suicídio durante o período faziam uso prejudicial de álcool e drogas. Tais dados foram coletados pelo Núcleo de Atenção ao Suicídio, do Instituto Philippe Pinel, na cidade do Rio de Janeiro, Brasil, no período de 2000 a 2002.

Finalmente, dados de centros de pesquisa indicam que as taxas de admissões hospitalares por tentativas de suicídio de adolescentes e adultos jovens cresceram rapidamente durante o mesmo período (1965 a 1980) em que suas taxas de suicídio estavam também aumentando (Diekstra, 1989).

Com base nessas observações e na relevância do tema, este trabalho teve como objetivos: 1) Analisar, comparativamente, a distribuição temporal de todos os casos de adolescentes com idade entre 10 e 24 anos, de ambos os sexos, atendidos no setor de urgências psiquiátricas de um hospital geral durante o período de 1988 a 2004, após tentativa de suicídio e após o uso/abuso de substâncias psicoativas; 2) Estudar a distribuição de todos os casos de adolescentes atendidos no setor, durante o período, após tentativas de suicídio, considerando as variáveis sexo, idade e método utilizado; 3) Apontar, por meio de um estudo de casos de adolescentes atendidos por tentativas de suicídio, dados sobre os motivos desencadeantes e sobre as histórias de vida desses pacientes, possivelmente relacionados com a ocorrência desse comportamento.

\section{Método}

A amostra estudada foi composta a partir de um levantamento retrospectivo de todos os casos de pacientes adolescentes atendidos no setor de urgências psiquiátricas do Hospital das Clínicas da Faculdade de Medicina de Ribeirão Preto da Universidade de São Paulo (FMRP-USP), durante o período de 1988 a 2004, após tentativa de suicídio e uso/abuso de substâncias psicoativas, e de dez histórias clínicas de casos de tentativas de suicídio de pacientes adolescentes selecionados ao acaso, atendidos no setor no ano de 1997. Dados incompletos não permitiram a inclusão dos anos de 1998 e 1999.

Os dados da investigação epidemiológica retrospectiva sobre as tentativas de suicídio e sobre os quadros relacionados ao uso de substâncias psicoativas foram fornecidos pelo serviço de estatística do hospital, codificados pela CID-9, de 1988 a 1997 (Organização Mundial da Saúde-OMS, 1979), e pela CID-10, a partir de 1998 (OMS, 1993). Os dados das histórias clínicas foram levantados por meio de entrevistas realizadas pela autora com os adolescentes e familiares, utilizando-se um formulário contendo questões relativas à identificação do paciente, sua história familiar, pessoal e social, e ao uso/abuso de substâncias psicoativas. Para esses casos, registros de atendimentos médicos também foram examinados. Para a classificação dos diagnósticos psiquiátricos dos adolescentes que tentaram o suicídio, foi adotado o que estabelece a classificação de transtornos mentais e de comportamento da CID-10. Entretanto, como a classificação utilizada para os diagnósticos psiquiátricos durante o período de 1988 a 1997 correspondia à da CID-9, foi feita a respectiva transposição da classificação dos diagnósticos utilizada naquele período para a adotada nesta investigação.

Foram considerados adolescentes todas as pessoas com idade entre 10 e 24 anos, procurando incluir 
as faixas apontadas pela Organização Mundial de Saúde, que considera o período de 10 a 20 anos, e por Setian, Colli \& Marcondes (1979), que introduzem o período de 15 a 25 anos.

Quanto à procedência, a grande maioria dos pacientes adolescentes atendidos no serviço era proveniente do município de Ribeirão Preto (75\%) e região (15\%), e somente uma minoria (10\%) era de outras regiões do país.

Para a análise da evolução temporal dos atendimentos durante o período, utilizou-se a regressão linear pelo método dos mínimos quadrados, e para o estudo da associação entre as variáveis, o método quiquadrado, para um nível de significância de 5\% ( $p=0,05)$.

Este projeto foi aprovado pelo Comitê de Ética em Pesquisa do Hospital das Clínicas da FMRP-USP, protocolo n॰ 950/98, e os pacientes ou os responsáveis (quando o paciente era menor de 18 anos) assinaram o termo de consentimento livre e esclarecido.

\section{Resultados}

Durante o período de 1988 a 2004, foram atendidos no setor de urgências psiquiátricas do
Hospital das Clínicas da FMRP-USP 1377 casos de tentativas de suicídio de adolescentes com idade entre 10 e 24 anos, sendo 1027 (75\%) do sexo feminino e 350 (25\%) do sexo masculino, o que estabelece uma relação aproximada de 3:1 em favor das mulheres.

No mesmo período, foram atendidos no setor 2271 casos de adolescentes, na mesma faixa etária, que apresentavam quadros clínicos relativos aos transtornos do uso de substâncias psicoativas, sendo 1911 (84\%) do sexo masculino e 358 (16\%) do sexo feminino - o que, portanto, estabeleceu uma relação aproximada de 5:1 em favor dos homens. Deste total, 949 (42\%) dos casos eram devidos ao uso de álcool (intoxicação aguda, síndrome de dependência, estado de abstinência, transtorno psicóticos) e 1320 (58\%) dos casos eram relacionados ao uso de outras drogas psicoativas, incluindo múltiplas drogas (intoxicação aguda, síndrome de dependência e transtorno psicótico devido, principalmente, ao uso de cocaína e maconha).

Verifica-se na Figura 1 que, enquanto proporcionalmente houve uma tendência significativamente ascendente a cada ano para os atendimentos por tentativas de suicídio ( $y=4,50+0,90 x, r=0,93, p=<0,0001)$ e por uso/abuso de substâncias psicoativas $(y=11,35+$

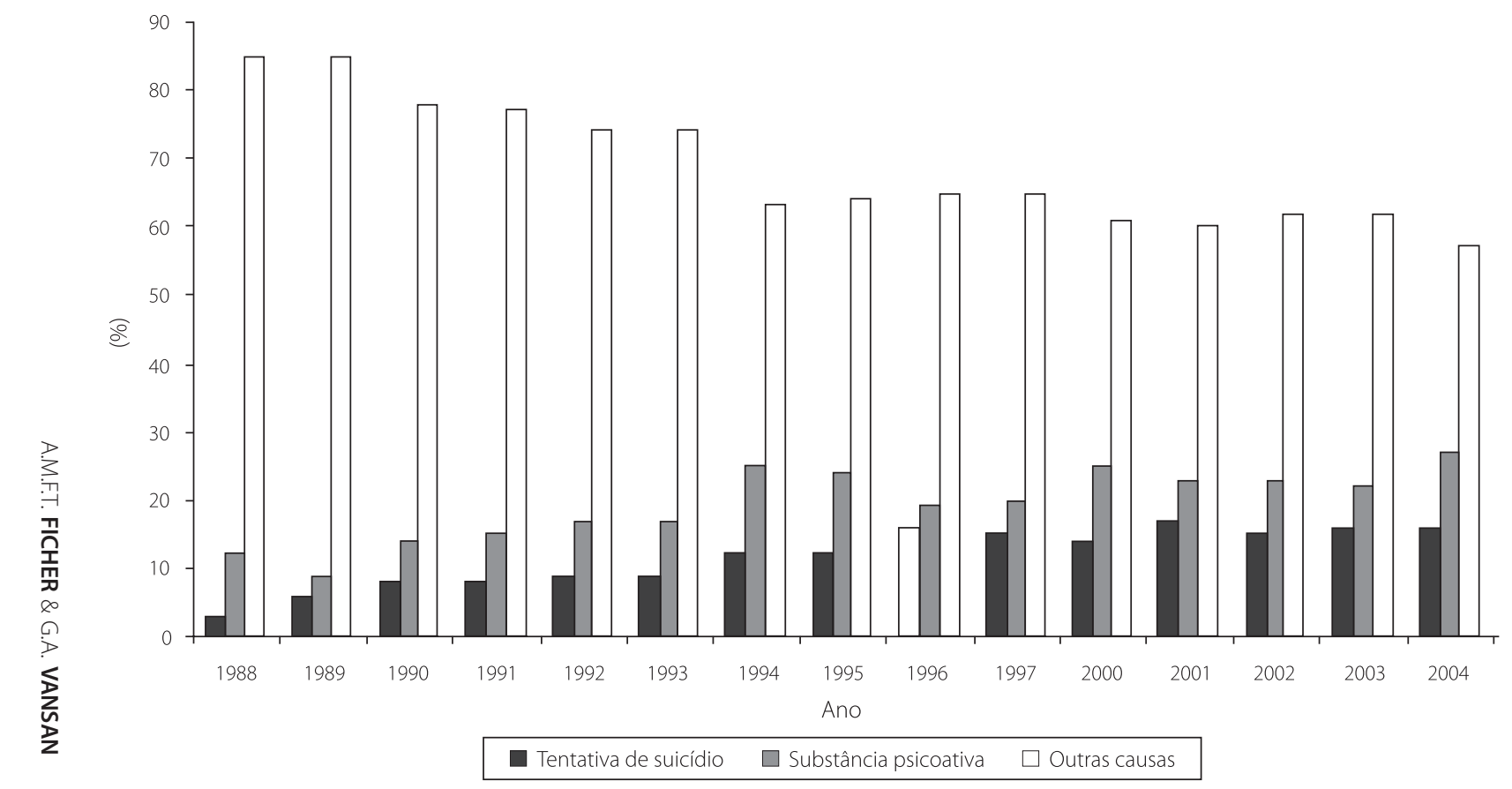

Figura 1. Distribuição percentual anual dos casos de adolescentes atendidos por tentativas de suicídio, por transtornos do uso de substâncias psicoativas, e por outras causas, no setor de urgências psiquiátricas do HCFMRP-USP, 1988-2004. 
$1,01 x, r=0,85, p=<0,0001)$, para os demais atendimentos houve uma tendência significativamente decrescente $(y=84,14-1,91 x, r=-0,93, p=<0,0001)$ durante o período.

Como é ilustrado na Figura 2, ocorreu, de maneira muito significativa durante o período, uma tendência crescente dos números de casos atendidos anualmente por tentativas de suicídio e por uso/abuso de substâncias psicoativas, tanto para os homens [(TS): $y=6,50+2,10 x, p=0,0005 ;(S P): y=75,34+6,05 x, p=0,0060]$, quanto para as mulheres [(TS): $y=28,58+4,98 x, p=0,0001$; (SP): $\mathrm{y}=12,61+1,40 \mathrm{x}, \mathrm{p}=0,0018]$.

Em relação ao início do período, esse crescimento foi, para as tentativas de suicídio, da ordem de 543\% (440\% para os homens e 590\% para as mulheres), e para o uso/abuso de substâncias psicoativas, da ordem de 151\% (respectivamente, 163\% e 107\%).

Os dados permitiram observar também, durante o período, uma forte correlação positiva entre os números de casos anualmente atendidos por tentativas de suicídio e os devidos ao uso/abuso de substâncias psicoativas, tanto para os adolescentes do sexo masculino $(r=0,70, p=0,0037)$, quanto para os do sexo feminino $(r=0,81, p=0,0002)$.

A distribuição das tentativas de suicídio em relação à idade permite observar, inicialmente, uma proporção mais elevada dos casos $(43,5 \%, \mathrm{n}=599) \mathrm{em}$ adolescentes com idades de 15 a 19 anos (Figura 3).

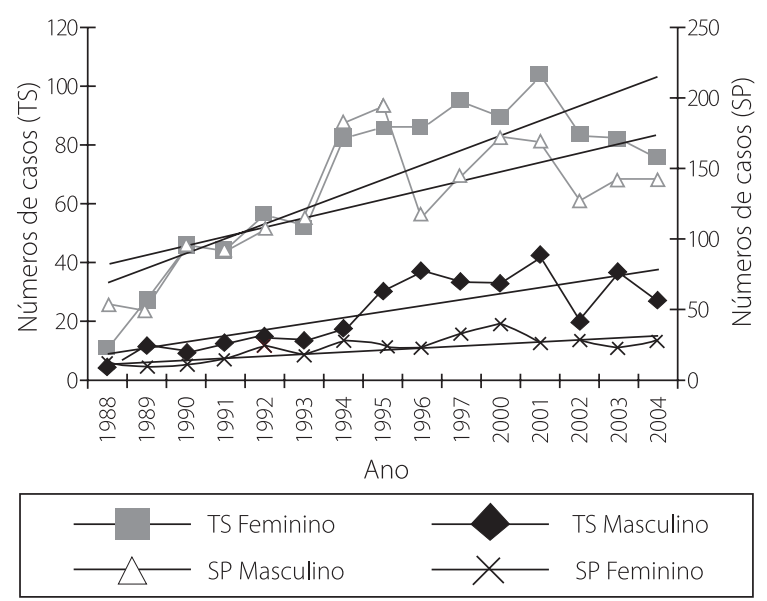

Figura 2. Distribuição numérica anual dos casos de tentativas de suicídio (TS) e de transtornos do uso de substâncias psicoativas (SP), de adolescentes, atendidos no setor de urgências psiquiátricas do HCFMRP-USP, 1988-2004.

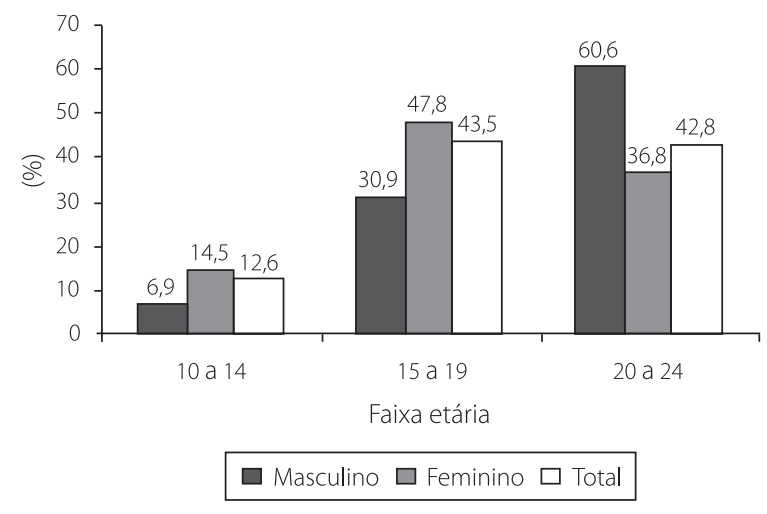

Figura 3. Distribuição percentual dos casos de tentativas de suicídio de adolescentes, segundo o sexo e a faixa etária, atendidos no setor de urgências psiquiátricas do HCFMRP-USP,1988-2004.

Quando considerada em relação ao sexo, verifica-se, de maneira significativa, que enquanto a grande maioria dos adolescentes do sexo masculino $(60,6 \%, n=212)$ encontra-se na faixa etária de 20 a 24 anos, uma freqüência mais elevada dos casos do sexo feminino $(47,8 \%, n=491)$ encontra-se em uma faixa um pouco mais jovem, constituída por adolescentes de 15 a $19 \operatorname{anos}\left(\chi^{2}=63,94, p<0,0001\right)$.

Verifica-se ainda que, proporcionalmente, as tentativas de suicídio das adolescentes na faixa dos 10 aos 14 anos foram duas vezes mais freqüentes que as dos adolescentes da mesma faixa etária. Ressalta-se que, no período, os números de tentativas de suicídio nas mulheres foram, em todas as faixas etárias, muito maiores que nos homens, na população estudada.

Conforme se observa na Figura 4, o método mais utilizado para as tentativas de suicídio foi, em ambos os sexos, o envenenamento (93,9\%, $n=1293)$, utilizando-se em ordem decrescente de importância a ingestão de medicamentos $(73,8 \%, n=1016)$ e a ingestão de outras substâncias químicas $(20,1 \%, n=277)$. 0 emprego de outros procedimentos ocorreu em apenas 6,1\% ( $n=84)$ dos casos, predominantemente com objetos cortantes, além de enforcamento (nos homens) e atear-se fogo (nas mulheres).

Verifica-se ainda, de maneira significativa, que enquanto a ingestão de medicamentos (psicofármacos e outros) foi mais freqüentemente empregada pelas mulheres $(79,8 \%)$ do que pelos homens $(56,0 \%)$, a ingestão de outras substâncias químicas, ao contrário, 


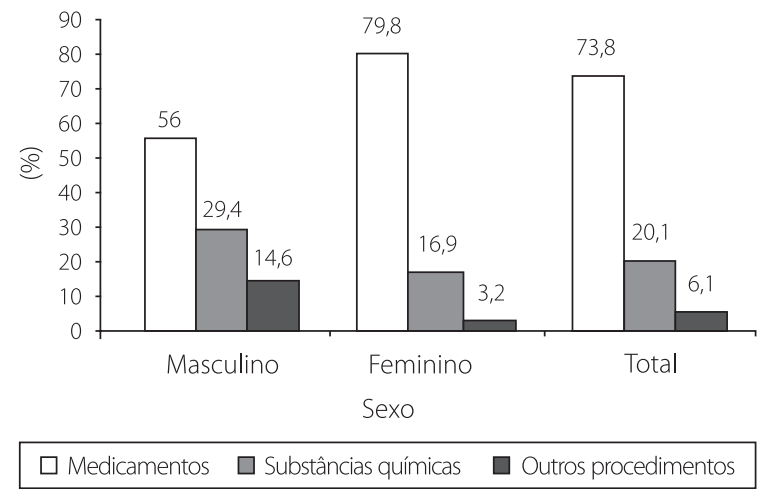

Figura 4. Distribuição percentual dos casos de tentativas de suicídio de adolescentes, segundo o sexo e o método utilizado, atendidos no setor de urgências psiquiátricas do HCFMRP-USP, 1988-2004. foi mais usada pelos homens $(44,0 \%)$ do que pelas mulheres, bem como outros procedimentos $(20,1 \%)$. $\left(\chi^{2}=95,5, p=<0,0001\right)$.

Para 692 casos de adolescentes com tentativas de suicídio (50\%), foram estabelecidos diagnósticos psiquiátricos (CID-10) (Tabela 1).

Os diagnósticos mais freqüentes em ordem decrescente de importância foram, no sexo feminino, os transtornos de ajustamento e outros transtornos neuróticos (39,2\%); os transtornos de personalidade $(28,1 \%)$, predominantemente a emocionalmente instável (15,6\%); os transtornos depressivos (14,1\%); as esquizofrenias (6,5\%); os transtornos mentais e de

Tabela 1. Distribuição dos diagnósticos psiquiátricos dos adolescentes com tentativas de suicídio atendidos no setor de urgências psiquiátricas do HCFMRP-USP, 1988 a 2004.

\begin{tabular}{|c|c|c|c|c|c|c|}
\hline \multirow{2}{*}{ Diagnósticos psiquiátricos } & \multicolumn{2}{|c|}{ Masculino } & \multicolumn{2}{|c|}{ Feminino } & \multicolumn{2}{|c|}{ Total } \\
\hline & $\mathrm{n}$ & $\%$ & $\mathrm{n}$ & $\%$ & $\mathrm{n}$ & $\%$ \\
\hline Transtorno de personalidade emocionalmente instável (borderline) & 15 & 6,5 & 72 & 15,6 & 87 & 12,6 \\
\hline Transtorno de personalidade não especificado & 27 & 11,7 & 49 & 10,6 & 76 & 11,0 \\
\hline Outros transtornos de personalidade & 5 & 2,2 & 9 & 1,9 & 14 & 2,0 \\
\hline Subtotal & 47 & 20,4 & 130 & 28,1 & 177 & 25,6 \\
\hline Episódio depressivo moderado & 2 & 0,9 & 14 & 3,0 & 16 & 2,3 \\
\hline Episódio depressivo grave sem sintomas psicóticos & 4 & 1,7 & 15 & 3,2 & 19 & 2,7 \\
\hline Episódio depressivo não especificado & 14 & 6,1 & 18 & 3,9 & 32 & 4,6 \\
\hline Episódio depressivo leve & 2 & 0,9 & 5 & 1,1 & 7 & 1,0 \\
\hline Episódio depressivo grave com sintomas psicóticos & 3 & 1,3 & 2 & 0,4 & 5 & 0,7 \\
\hline Transtornos do humor (afetivos) não especificados & 4 & 1,7 & 4 & 0,9 & 8 & 1,2 \\
\hline Outros transtornos do humor (afetivos) & 3 & 1,3 & 7 & 1,5 & 10 & 1,4 \\
\hline Subtotal & 32 & 14,0 & 65 & 14,1 & 97 & 14,0 \\
\hline Transtornos de ajustamento & 40 & 17,4 & 117 & 25,3 & 157 & 22,7 \\
\hline Outros transtornos neuróticos, relacionados ao estresse e somatoformes & 13 & 5,7 & 64 & 13,9 & 77 & 11,1 \\
\hline Subtotal & 53 & 23,0 & 181 & 39,2 & 234 & 33,8 \\
\hline Transtornos mentais e de comportamento decorrentes do uso de álcool & 28 & 12,2 & 9 & 1,9 & 37 & 5,3 \\
\hline $\begin{array}{l}\text { Transtornos mentais e de comportamento decorrentes do uso de outras substâncias } \\
\text { psicoativas }\end{array}$ & 22 & 9,6 & 10 & 2,2 & 32 & 4,6 \\
\hline Subtotal & 50 & 21,7 & 19 & 4,1 & 69 & 10,0 \\
\hline Esquizofrenias & 33 & 14,3 & 27 & 5,8 & 60 & 8,7 \\
\hline Transtorno esquizoafetivo, tipo depressivo & & & 2 & 0,4 & 2 & 0,3 \\
\hline Psicose não-orgânica não especificada & 3 & 1,3 & 1 & 0,2 & 4 & 0,6 \\
\hline Subtotal & 36 & 15,7 & 30 & 6,5 & 66 & 9,5 \\
\hline Transtornos de conduta & 2 & 0,9 & 16 & 3,5 & 18 & 2,6 \\
\hline Transtornos mistos de conduta e emoções & 1 & 0,4 & 1 & 0,2 & 2 & 0,3 \\
\hline Subtotal & 3 & 1,3 & 17 & 3,7 & 20 & 2,9 \\
\hline Transtorno mental não especificado & & & 5 & 1,1 & 5 & 0,7 \\
\hline Outros transtornos mentais e de comportamento & 9 & 3,9 & 15 & 3,2 & 24 & 3,5 \\
\hline Subtotal & 9 & 3,9 & 20 & 4,3 & 29 & 4,2 \\
\hline Total & 230 & 100,0 & 462 & 100,0 & 692 & 100,0 \\
\hline
\end{tabular}


comportamento decorrentes do uso de substâncias psicoativas (4,1\%); e os transtornos de conduta (3,7\%). As três primeiras categorias correspondem aos diagnósticos de $81,4 \%$ dos casos.

No sexo masculino, os diagnósticos mais freqüentes foram os transtornos de ajustamento e outros transtornos neuróticos (23,0\%); os transtornos mentais e de comportamento decorrentes do uso de substâncias psicoativas (21,7\%), predominantemente devido ao uso de álcool (12,2\%); os transtornos de personalidade $(20,4 \%)$; as esquizofrenias $(15,7 \%)$; e os transtornos depressivos (13,9\%).

Quanto aos casos clínicos, foram entrevistados sete adolescentes do sexo feminino e três do masculino, segundo a seguinte distribuição etária: uma adolescente com 13 anos, três mulheres e um homem na faixa etária de 15 a 19 anos, e três mulheres e dois homens na faixa etária de 20 a 24 anos. Outros aspectos relevantes das suas histórias clínicas são apresentados na discussão dos resultados.

\section{Discussão}

Os estudos epidemiológicos das tendências evolutivas dos comportamentos suicidas, particularmente das tentativas de suicídio, contam com numerosas dificuldades metodológicas, derivadas principalmente das fontes de obtenção dos dados, que freqüentemente levantam dúvidas quanto à generalização de seus resultados. A maioria das pesquisas para monitorar as tendências relativas aos atos suicidas não fatais tem sido baseada em dados de registros hospitalares de pacientes admitidos em serviços de urgências de hospitais gerais e, obviamente, o fenômeno ocorre mais amplamente do que este procedimento sugere.

Entretanto, sem ignorar os problemas metodológicos existentes e o fato de se estar considerando apenas os atendimentos do setor de urgências psiquiátricas de um grande hospital público, é razoável supor que o crescente aumento no número de admissões hospitalares observado durante o período reflete um crescimento desse comportamento na população geral, ou indica, ao menos, que esse aumento evidencia atualmente um sério problema médico- psicológico, em nível assistencial, entre os adolescentes e adultos jovens.

O mesmo pode-se dizer em relação ao crescente aumento dos atendimentos a adolescentes e adultos jovens usuários de substâncias psicoativas, no mesmo período.

Furtado (1998) apontou, na Unidade de Emergência do Hospital das Clínicas da FMRP-USP, no período de 1987 a 1994, um crescimento nos atendimentos a casos de crianças, adolescentes e jovens até 24 anos, com quadros envolvendo diagnósticos relacionados ao uso, abuso e dependência de substâncias psicoativas, sendo a maioria de drogas que não o álcool.

Pode-se depreender dessas observações que as taxas de prevalência do consumo de drogas no município também cresceram durante o período, provocando um crescente aumento da demanda aos serviços de saúde por parte da população como um todo, mas, principalmente, por adolescentes e adultos jovens do sexo masculino, populações mais vulneráveis ao uso/abuso e dependência de substâncias psicoativas.

Alguns fatos corroboram este dado: no período de 1994 a 1997 ocorreu no município um aumento progressivo no número de apreensões envolvendo porte e tráfico de drogas ilícitas, passando de 348 em 1994 a 1108 apreensões em 1997. Considerando o índice de apreensões de 5\% (5\% a 10\% das drogas produzidas no mundo são apreendidas pela polícia) e o volume de mercadoria apreendida, conclui-se que o comércio de drogas em Ribeirão Preto e nos municípios vizinhos movimenta quase R\$ 100 milhões. Ao mesmo tempo, o tráfico de drogas elevou em muito os índices de violência. Dados procedentes da Delegacia Seccional de Ribeirão Preto mostraram que, de 1995 para 1997, o número de homicídios subiu de 96 para 209 e, desse total, 85\% das vítimas tinham antecedentes criminais quase sempre ligados ao tráfico de drogas (Furtado, 1998).

Atenta-se então, para as conseqüências médicas e psicossociais negativas que esses fatos acarretam na população, especialmente entre os adolescentes e adultos jovens. É na adolescência que o uso/abuso de substâncias psicoativas (álcool e drogas) tem despertado uma preocupação maior em profissionais da área de saúde, uma vez que a primeira experiência com estas 
substâncias ocorre freqüentemente nesta fase do desenvolvimento (Muza, Bettiol, Muccillo \& Barbieri, 1997).

Muito mais freqüente do que o consumo de drogas é o consumo de bebidas alcoólicas entre os adolescentes e jovens, principalmente entre os homens.

Muza et al. (1997), estudando, em 1990, o consumo de substâncias psicoativas por adolescentes de 13 a 19 anos, da oitava série do primeiro grau e primeiro, segundo e terceiro anos do segundo grau, de escolas públicas e privadas do município de Ribeirão Preto, encontraram para o álcool uma taxa de prevalência de uso, na vida de $88,9 \%$, no último ano de $80,7 \%$, no último mês de 56,4\%, e diário de 8,5\%. Essas taxas, mesmo podendo não refletir a verdadeira magnitude do problema na população geral de adolescentes, cuja realidade é muito diferente da de escolares, mostram que no município a prevalência de consumo de bebidas alcoólicas nesta fase do desenvolvimento é bastante alta e preocupante.

É relevante considerar, nesse sentido, que os resultados de dois levantamentos domiciliares sobre o uso de drogas psicotrópicas no Brasil, realizados em 2001 (Carlini, Galduróz, Noto \& Nappo, 2002) e 2005 (Carlini, 2006), mostram o consumo de bebidas alcoólicas em faixas etárias cada vez mais precoces e, comparativamente, um crescimento das taxas de prevalência de uso na vida e de dependência do álcool, bem como de problemas relacionados com a bebida, na população de adolescentes e adultos jovens, respectivamente, com idades de 12 a 17 e 18 a 24 anos. Fato preocupante é a constatação, em ambos os estudos, de que no Brasil o maior número de casos de dependentes de bebidas alcoólicas encontra-se entre os homens na faixa etária dos 18 aos 24 anos (taxa de prevalência de 23,7\% em 2001 e 27,4\% em 2005). Dada a estreita relação existente entre o uso abusivo de drogas e o de álcool (Lopes, Mari \& Warcwald, 1991), é plausível supor, pelas razões expostas anteriormente, que a prevalência crescente do consumo de drogas nos últimos anos, nesta população, tenha provocado também um crescimento da demanda aos serviços de saúde por parte de adolescentes usuários de álcool.

Por outro lado, estudos têm demonstrado existir entre os adolescentes e adultos jovens usuários de substâncias psicoativas uma associação importante entre abuso/dependência de drogas e/ou álcool e morbidade psiquiátrica, principalmente depressão (Lopes et al., 1991), bem como entre essas substâncias e comportamentos suicidas (ideação suicida, tentativas de suicídio e suicídios completos) (Crumley, 1982).

Crumley (1990), em uma revisão da literatura sobre abuso de substâncias psicoativas e comportamentos suicidas, aponta evidências cumulativas (embora não consistentes) em todos os trabalhos, sugerindo uma significativa associação entre o aumento da incidência dos transtornos do uso de substâncias psicoativas e o aumento da incidência de suicídios e tentativas de suicídio entre os adolescentes nas duas décadas anteriores.

Dados de pesquisas sobre a ocorrência desses comportamentos nessas duas décadas, nos Estados Unidos, mostraram que as porcentagens de adolescentes vítimas de suicídios e tentativas de suicídio que abusavam de drogas psicoativas cresceram acentuadamente durante o período (Crumley, 1990). Entre outros aspectos, Crumley conclui que os trabalhos revisados mostram evidências crescentes indicando uma associação significativa entre abuso de substâncias psicoativas e uma maior incidência e mais repetições de tentativas de suicídio, tentativas com intenção suicida e letalidade médica aumentadas, e ideação suicida aumentada.

Ainda que vários estudos sustentem uma relação direta entre o abuso de substâncias psicoativas e comportamentos suicidas, principalmente entre jovens, a natureza desta relação não está claramente estabelecida, e requer mais investigações.

Entretanto, independentemente deste conhecimento, este e outros estudos (Borges, Walters \& Kessler, 2000; Thatcher, Reininger \& Drane, 2002) sugerem fortemente que o uso/abuso/dependência de substâncias psicoativas devem ser considerados como um importante fator de risco para a ocorrência de comportamentos suicidas de adolescentes.

No estudo aqui relatado, entrevistou-se uma amostra casualmente composta por dez adolescentes que tentaram o suicídio durante o ano de 1997, admitidos no setor de urgências psiquiátricas do Hospital das Clínicas de Ribeirão Preto.

Dessa amostra, composta por três homens e sete mulheres com idades entre 10 e 24 anos, três pacientes (30\%, sendo dois homens e uma mulher) revelaram em 
suas histórias clínicas fazer uso abusivo de substâncias psicoativas (maconha e cocaína), e um paciente, embora negasse seu uso, era suspeito de envolvimento com drogas, segundo declaração dos pais.

Apesar do pequeno número de casos, este resultado (30\% a 40\%), diferentemente do que foi observado com os dados sobre tentativas obtidos retrospectivamente para o período em estudo (10\%, Tabela 1), é semelhante aos encontrados em vários dos trabalhos revisados por Crumley (1990), mostrando um aumento da incidência de ambas as condições entre os adolescentes.

Apesar das suas limitações, os resultados obtidos neste trabalho e, paralelamente, o crescimento do consumo de drogas no município nos últimos anos, sugerem a associação entre uso/abuso de drogas e tentativas de suicídio, muito significativa em nosso meio, e ressaltam a importância de mais pesquisas com o objetivo de conhecer a magnitude e a natureza deste problema, que pode ser considerado de saúde pública, uma vez que a tentativa de suicídio é um dos maiores preditores de suicídio, e que 10\% a 14\% das pessoas envolvidas em tentativas de suicídio eventualmente o completam, um risco 100 vezes maior que para a população geral (Diekstra, 1993).

Quanto aos demais resultados obtidos, eles permitem observar, inicialmente, que as tentativas de suicídio nos adolescentes atendidos ocorreram com uma freqüência significativamente mais elevada nas mulheres, predominantemente em uma faixa etária (15 a 19 anos) mais jovem do que a de maior freqüência nos homens (20 a 24 anos), em uma razão aproximada de 3:1 em favor das mulheres.

Embora o grupo etário de maior incidência, em ambos os sexos, possa variar entre países ou regiões, constata-se em épocas e locais diversos que a tentativa de suicídio não está preferencialmente circunscrita ao adulto, mas, ao contrário, ela representa um problema maior entre os adolescentes e adultos jovens, sendo mais comum nas mulheres em idades mais jovens do que as de maiores freqüências nos homens (Avanci et al., 2005; Hawton \& Catalan, 1987a; Schmidtke et al., 1996; Vansan, 1999; Werneck et al., 2006).

Nesse sentido, os estudos mostram que, em ambos os sexos, as taxas de tentativas de suicídio diminuem substancialmente na meia idade, e são extremamente baixas após os 55 ou 60 anos (Andrade, 1979; Hawton \& Catalan, 1987b; Schmidtke et al., 1996) e, de igual modo, indicam que, em números, a ocorrência desse comportamento é decrescente com a idade, sendo muito menor a partir dos 50 ou 55 anos (Andrade, 1979; Schmidtke et al., 1996; Vansan, 1999). Em geral, pode-se dizer que os atendimentos às tentativas de suicídio dos mais velhos representam somente em torno de $5 \%$ do total dos casos de tentativas atendidas nos serviços de urgência (García-Portilha, Martinez, Garcia \& Corujo, 1997).

Entretanto, é importante ressaltar que as tentativas que ocorrem entre as pessoas de mais idade constituem um grupo particular, cujas características se aproximam mais às dos suicídios completos do que às das próprias tentativas. Assim, qualquer gesto de intencionalidade autodestrutiva nessas pessoas, por menor que seja, deve ser considerado com absoluta seriedade, uma vez que as tentativas entre elas terminam com mais freqüência em um ato suicida fatal (Garcia-Portilha et al., 1997).

Assim, dando suporte aos resultados aqui obtidos, os estudos mostram de maneira consistente que a tentativa de suicídio é mais comum em jovens, e que adolescentes, especialmente mulheres, formam uma proporção considerável de pacientes admitidos em hospitais gerais, após seus atos suicidas não fatais.

As razões pelas quais as mulheres realizam mais tentativas de suicídio do que os homens são motivos de numerosas especulações. No entanto, é plausível supor que a utilização significativamente mais freqüente pelas mulheres do que pelos homens de métodos menos violentos, como medicamentos, deve contribuir para uma proporção mais elevada de mulheres que tentam o suicídio. Ao contrário, o emprego mais freqüente de métodos violentos pelos homens, isto é, aqueles que levam a tentativas de suicídio com menor probabilidade de salvamento, deve ser um fator para uma proporção maior de homens que completam o suicídio.

A depressão é significativamente mais comum nas mulheres do que nos homens que, em contraste, apresentam mais comumente distúrbios que envolvem comportamentos agressivos e violentos e problemas com impulsividades (Newson-Smithe \& Hirsche, 1979). Assim, é plausível supor que a mulher, sendo mais inclinada a comunicar seus sentimentos aos outros, use 
a tentativa de suicídio como forma de expressão de sua tristeza e como um pedido de ajuda de maneira mais freqüente do que o homem. Este, por sua vez, sendo menos inclinado a comunicar sentimentos, defende-se contra os sentimentos de tristeza e impotência por meio do uso/abuso de substâncias psicoativas, que podem agravar o risco das tentativas de suicídio devido às perturbações físicas, sociais e interpessoais severas desencadeadas pelas mesmas (Diekstra, 1993).

É relevante observar que, atualmente, em muitos países industrializados do mundo, a maior prevalência de transtornos depressivos está entre os adolescentes e adultos jovens, e que, nas últimas décadas, a idade de início do uso/abuso de substâncias psicoativas tem declinado paralelamente com a diminuição da idade de início dos transtornos depressivos (Diekstra, 1993).

Esses fatos têm explicado as tendências crescentes das taxas de suicídio e tentativas de suicídio que ocorreram em vários países do mundo entre os adolescentes e adultos jovens, nas últimas décadas. Em nosso meio, pelo que já foi exposto anteriormente em relação ao aumento de consumo de álcool e drogas, bem como sua estreita relação com os transtornos depressivos, esses fatos já devem estar presentes como fatores de risco suicida entre os adolescentes, em um grau consideravelmente elevado.

A propósito, Crumley (1982) aponta as síndromes depressivas, com grande diferença, como os transtornos observados com mais freqüência nos adolescentes com tentativas de suicídio, seguidos da dependência do álcool e drogas.

Em concordância com resultados já amplamente conhecidos da literatura (Andrade, 1979; Avanci et al., 2005; Freitas, Rapeli \& Botega, 2004; Marcondes Filho et al., 2002; Schmidtke et al., 1996), o método mais utilizado pelos adolescentes em suas tentativas de suicídio foi, em ambos os sexos, o envenenamento ( $93,9 \%$ dos casos), predominantemente por meio da ingestão de medicamentos $(73,8 \%)$, em uma freqüência mais elevada nas mulheres $(79,8 \%)$ do que nos homens $(56,0 \%)$. É fato também que a maioria das tentativas de suicídio com medicamentos envolve drogas prescritas, sendo os tranqüilizantes menores e os sedativos os mais comumente utilizados (Hawton \& Catalan, 1987a; Vansan, 1999).

Os resultados do estudo aqui descrito e de outras 370 pesquisas que estudaram pacientes admitidos em hospitais gerais após suas tentativas de suicídio mostram que os psicofármacos, predominantemente os tranqüilizantes menores, apesar de ocuparem uma posição de destaque como agentes autodestrutivos, não foram os medicamentos mais utilizados pelos adolescentes. Nesses casos foi usada de forma mais freqüente uma ampla gama de outros medicamentos, que incluem desde drogas que não requerem prescrições médicas, como os analgésicos (aspirina, paracetamol), até aquelas que comumente envolvem prescrições, como os anticonvulsivantes, antialérgicos, antibióticos e várias outras drogas não psicotrópicas (Hawton \& Catalan, 1987a; Vansan, 1999).

Dados dos últimos cinco anos do período, atualmente classificados pela CID-10 em uma única categoria (X61, que inclui as drogas antiparkinsonianas, anti-epilépticas, os barbitúricos, os tranqüilizantes e os antidepressivos, que não são rotineiramente discriminados nas estatísticas hospitalares) mostram que, nesse período, o procedimento mais utilizado pelas mulheres em suas tentativas de suicídio foi a ingestão desses medicamentos (mais freqüentemente os tranqüilizantes menores), e pelos homens a ingestão de outras substâncias químicas.

É importante ressaltar que quando drogas prescritas são usadas, e isto é particularmente verdadeiro para os agentes psicotrópicos e os tranqüilizantes menores, muito freqüentemente elas foram prescritas para o paciente um pouco antes do ato suicida não fatal, momento em que o mesmo se encontrava em estado de ansiedade ou depressão, associado com dificuldades interpessoais e sociais (Hawton \& Catalan, 1987b). Nesse sentido, há evidências de uma nítida correlação entre o crescimento de taxas de tentativa de suicídio por envenenamento e o aumento da prescrição de drogas psicotrópicas e, igualmente, um declínio nas taxas de tentativas por esse procedimento, em paralelo à diminuição da prescrição desses medicamentos, no mesmo período de tempo (Hawton \& Catalan, 1987a). 0 papel significativo dos medicamentos nas tentativas de suicídio é indicado também por Cassorla (1981), ao observar que comumente as drogas utilizadas nas tentativas por ele investigadas estavam sendo tomadas pelos suicidas ou parentes e, algumas vezes, foram compradas especialmente para o ato autodestrutivo. 
Portanto, todos os estudos, incluindo o mais paradigmático sobre o tema atualmente, o WHO/EURO multicentre study on parasuicide, indicam que a intoxicação medicamentosa é o procedimento mais utilizado por aqueles que tentam o suicídio, sendo usado, entretanto, mais freqüentemente pelas mulheres (Schmidtke et al., 1996), em concordância com os resultados aqui obtidos.

Por outro lado, tem-se observado que a escolha de um método suicida tem uma estreita relação com sua disponibilidade, aceitação sociocultural e letalidade (Cantor, 1989). Nesse sentido, Lester (1989) relata uma diminuição nas taxas de suicídio em alguns países após a limitação da disponibilidade de algumas substâncias. Sendo assim, cuidadosas precauções na prescrição de medicamentos e a adoção de rigorosas medidas para seu controle na comunidade podem contribuir para a prevenção de comportamentos suicidas.

Quanto aos quadros clínicos apresentados por esses adolescentes, é importante considerar que, dada a natureza transitória de muitos dos sintomas comumente apresentados por eles, é difícil saber ao certo quantos têm um transtorno psiquiátrico "definido".

Embora sintomas psiquiátricos sejam comuns (humor deprimido, tensão nervosa, desesperança, irritabilidade, aborrecimento, baixa concentração), a proporção de pacientes com diagnóstico psiquiátrico estabelecido é variável, e depende também do momento escolhido para se realizar a avaliação.

Dois estudos realizados com pacientes de todas as idades que tentaram o suicídio por envenenamento e foram avaliados por meio do Present State Examination (PSE) apontaram índices compatíveis com transtornos psiquiátricos "definidos" em 31\% dos casos (NewsonSmithe \& Hirsch, 1979) e 27\% (Urwin \& Bibbons, 1979). Adicionalmente, mais 29\% e $42 \%$ dos casos, respectivamente, estavam na categoria limítrofe ou borderline, isto é, eram pacientes com transtornos menos severos, classificados no nível inferior dos índices de sintomas compatíveis com transtornos psiquiátricos. A maioria dos pacientes de ambos os estudos foi diagnosticada como portadora de um transtorno depressivo. Avaliados novamente uma semana e três meses após a ocorrência das tentativas, as proporções de pacientes com transtornos psiquiátricos classificados em ambas as categorias (31\% e 29\%) decresceram progressivamente (Newson-Smithe \& Hirsch, 1979).
Assim, pode-se concluir que, para uma parcela considerável dos casos, os sintomas indicam transtornos psiquiátricos, que em geral apresentam um perfil de sintomatologia neurótica, de intensidade leve ou moderada, com características depressivas e que, em sua maioria, são também transtornos relativamente transitórios e, ao que parece, usualmente secundários a eventos estressantes freqüentemente ocorridos na vida dos pacientes pouco antes dos seus atos suicidas (Hawton \& Catalan, 1987b).

Ainda que não se tenha o registro desses fatos, é bastante plausível sugerir que os diagnósticos de transtornos de ajustamento, outros transtornos neuróticos e episódios depressivos leves e moderados apresentados por 37\% da amostra dos adolescentes aqui avaliados estariam incluídos nesses casos. Entrevistas realizadas com a amostra de dez desses adolescentes, logo após suas tentativas de suicídio, sustentam, em parte, essa observação. A propósito, o DSM-1 classificava todas as reações de ajustamento sob o título de "Transtornos de personalidade situacionais transitórios" (Wise, 1992).

Quando se analisa as histórias de vida desses jovens verifica-se que, em geral, eles são provenientes de lares desestruturados ou "lares desfeitos" (broken homes). Isto significa que a maioria sofreu durante a infância a perda de um ou de ambos os progenitores, por morte, separação/divórcio ou abandono (Cassorla, 1981; Dorpat, Jakson \& Ripley, 1965; Greer, 1964; Hawton \&Catalan, 1987b).

Neste trabalho, observou-se que sete (70\%) dos adolescentes que tentaram suicídio provinham de"lares desfeitos", isto é, haviam sofrido perdas parentais em fases precoces do desenvolvimento. Para cinco desses casos, as perdas ocorreram por separação dos pais, nas idades entre 3 e 10 anos, e para dois casos por morte do pai, sendo uma por suicídio (respectivamente, nas idades de 3 e 14 anos). Assim, embora seja pequeno o número de casos estudados, os dados parecem sugerir, em concordância com as observações anteriores, que há uma forte associação entre uma história de perda parental na infância, predominantemente por separação dos pais, e tentativa de suicídio, entre os adolescentes e adultos jovens. Dorpat et al. (1965), examinando esta condição entre os que tentam e os que completam o suicídio, aventou a hipótese de que "as perdas objetais não resolvidas na infância levariam a uma incapacidade 
para suportar perdas objetais posteriormente na vida; isto, por sua vez, levaria a reações depressivas que culminariam no comportamento suicida".

Entrevistando essa pequena amostra de pacientes, observou-se que esses jovens se sentiam desvalorizados, rejeitados e desprezados pelas pessoas dos seus núcleos sócio-familiares, e apresentavam intensos e constantes problemas de relacionamento com suas famílias de origem e/ou com seus respectivos parceiros. Esses problemas, que envolviam comumente brigas e discussões dos adolescentes com alguma pessoa significativa dos seus relacionamentos (mãe, irmãos, parentes, namorado(a), cônjuge), foram, em todos os casos, os motivos determinantes das suas tentativas de suicídio, e na maioria dos casos tais brigas ou discussões ocorreram próximo ao momento do ato suicida. Separações e ameaças de separações, reais ou imaginárias, daquelas pessoas significativas das relações sócio-familiares, integram o conjunto de motivos para a tentativa de suicídio. Botega, Cano e Kohn (1995) também encontraram como principais motivações para as tentativas de suicídio a briga recente do suicida com uma pessoa próxima do seu relacionamento e os problemas amorosos.

Quando se acrescenta às perdas parentais reais precoces aquelas determinadas por separações psicológicas, isto é, situações nas quais a criança sente-se isolada dos pais devido à rejeição ou à indiferença dos mesmos, mesmo na presença deles, a dificuldade de lidar com a separação na infância será acentuadamente elevada, e a isto se deve, em parte, o aumento da freqüência de jovens com maior vulnerabilidade a separações posteriormente na vida. Assim, embora sejam necessárias novas investigações, os dados parecem sugerir que experiências de perdas parentais na infância, predominantemente por separação/divórcio, e muito provavelmente também as psicológicas, devem ser consideradas como um importante fator de risco para a ocorrência de tentativas de suicídio, uma vez que tais situações levariam a uma sensibilidade maior desses jovens às separações ou ameaças de separações futuras, que são condições freqüentemente associadas à ocorrência desse comportamento.

Cabe destacar, finalmente, que os adolescentes entrevistados, em sua maioria, relataram que comunicaram suas intenções suicidas diretamente às pessoas de seus núcleos sócio-familiares anteriormente ao comportamento auto-destrutivo. Alguns relataram pretender repetir o comportamento posteriormente, procurando uma maneira mais eficaz, para que tenham êxito em suas tentativas. Essas comunicações, que também ocorrem entre os que completam o suicídio (Vansan \& Fávero, 1987), deveriam ser compreendidas como verdadeiros pedidos de ajuda, e como uma importante oportunidade para a prevenção de tais comportamentos.

\section{Considerações Finais}

Os resultados desta investigação mostraram que, durante o período de 1988 a 2004, foram atendidos no setor de urgências psiquiátricas do HCFMRP-USP 1377 casos de tentativas de suicídio de adolescentes com idades entre 10 e 24 anos, sendo $75 \%$ do sexo feminino e $25 \%$ do sexo masculino, o que estabelece uma relação aproximada de 3:1 em favor das mulheres.

No mesmo período, foram atendidos 2271 casos de adolescentes, na mesma faixa etária, apresentando quadros clínicos relativos aos transtornos do uso/abuso de substâncias psicoativas, sendo 84\% do sexo masculino e $16 \%$ do sexo feminino e, portanto, estabelecendo uma relação aproximada de 5:1 em favor dos homens.

Encontrou-se de maneira muito significativa, durante o período, uma tendência crescente dos números de casos atendidos anualmente por tentativas de suicídio e por uso/abuso de substâncias psicoativas, tanto para os homens, quanto para as mulheres.

Encontrou-se também uma forte correlação positiva, durante o período, entre os números de casos anualmente atendidos por tentativas de suicídio e por uso/abuso de substâncias psicoativas, tanto para os adolescentes $(r=70, p=0,0037)$ quanto para as adolescentes $(r=0,81, p=0,0002)$.

A distribuição dos casos de adolescentes atendidos no setor de urgências psiquiátricas do HCFMRPUSP por tentativas de suicídio mostra, de maneira significativa, que enquanto a grande maioria das tentativas entre os homens $(60,6 \%)$ ocorreu na faixa etária de 20 a 24 anos, as das mulheres ocorreram com uma freqüência mais elevada $(47,8 \%)$ em uma faixa um pouco mais jovem, constituída por adolescentes de 15 a 19 $\operatorname{anos}\left(\chi^{2}=63,94, p<0,0001\right)$. 
O método mais utilizado para as tentativas de suicídio foi, em ambos os sexos, o envenenamento (93,9\%), utilizando-se em ordem decrescente de importância a ingestão de medicamentos (73,8\%) e a ingestão de outras substâncias químicas (20,1\%). O emprego de outros procedimentos ocorreu em apenas 6,1\% dos casos. Enquanto a ingestão de medicamentos (psicofármacos e outros) foi mais freqüentemente empregada pelas mulheres (79,8\%) do que pelos homens (56,0\%), a ingestão de outras substâncias químicas, ao contrário, foi mais usada pelos homens (44,0\%) do que pelas mulheres, bem como outros procedimentos $(20,1 \%)\left(\chi^{2}=95,5, p<0,0001\right)$.

Os diagnósticos clínicos dos adolescentes que tentaram suicídio foram, em ordem decrescente de importância no sexo feminino, os transtornos de ajustamento e outros transtornos neuróticos; os transtornos de personalidade, predominantemente a emocionalmente estável; os transtornos depressivos; as esquizofrenias; os transtornos decorrentes do uso de substâncias psicoativas; e os transtornos de conduta. No sexo masculino, os diagnósticos mais freqüentes, em ordem decrescente de importância, foram os transtornos de ajustamento e outros transtornos neuróticos; os transtornos decorrentes do uso de substâncias psicoativas, predominantemente devido ao uso de álcool; os transtornos de personalidade; as esquizofrenias; e os transtornos depressivos.

As histórias clínicas dos pacientes entrevistados mostraram que a grande maioria deles (70\%) provinha de lares desfeitos, isto é, eram jovens que haviam sofrido perdas parentais em fases precoces de desenvolvimento, sendo a maioria por separação dos pais. Além disso, eram adolescentes que se sentiam intensamente desvalorizados, rejeitados e desprezados pelas pessoas dos seus núcleos sócio-familiares, e que apresentavam intensos e constantes problemas de relacionamentos familiares e amorosos, que foram os motivos determinantes de suas tentativas. $\mathrm{O}$ uso/abuso de substâncias psicoativas esteve presente em 40\% dos casos estudados.

\section{Referências}

Andrade, J. J. B. (1979). Epidemiologia da tentativa de suicídio em Ribeirão Preto. Dissertação de mestrado não-publicada, Faculdade de Medicina de Ribeirão Preto, Universidade de São Paulo.
Avanci, R. C., Pedrão, L. J., \& Costa Júnior, M. L. (2005). Perfil do adolescente que tenta suicídio em uma unidade de emergência. Revista Brasileira de Enfermagem, 58 (5), 535-539.

Borges, B., Walters, E. E., \& Kessler, R. (2000). Association of substance use, abuse, and dependence with subsequent suicidal behavior. American Journal of Epidemiology, 151 (8), 781-789.

Botega, N. J., Cano, F., \& Kohn, S. L. (1995). Tentativa de suicídio e adesão ao tratamento: um estudo descritivo em hospital geral. Jornal Brasileiro de Psiquiatria, 44 (1), 19-25.

Cantor, C. H. (1989). Substance involved in fatal drug overdoses in Briskane, 1979-1987. Acta Psychiatrica Scandinavica, 80 (Suppl. 354), 69-71.

Carlini, E. A. (2006). Il levantamento domiciliar sobre o uso de drogas psicotrópicas no Brasil: estudo envolvendo as 108 maiores cidades do país - 2005. São Paulo: Centro Brasileiro de Informações sobre Drogas Psicotrópicas.

Carlini, E. A., Galduróz, J. C. F., Noto, A. R., \& Nappo, A. S. (2002). I levantamento domiciliar sobre o uso de drogas psicotrópicas no Brasil - 2001. São Paulo: Centro Brasileiro de Informações sobre Drogas Psicotrópicas.

Cassorla, R. M. S. (1981). Jovens que tentam o suicídio. Tese de doutorado não-publicada, Faculdade de Ciências Médicas, Universidade Estadual de Campinas.

Cassorla, R. M. S. (1992). O que é suicídio (Coleção Primeiros Passos, $4^{a}$ ed.) São Paulo: Brasiliense.

Crumley, F. E. (1982). The adolescent suicide attemp: a cardinal symptom of a serious psychiatric disorder. American Journal of Psychoterapy, 36, 155-65.

Crumley, F. E. (1990). Substance abuse and adolescent suicidal behavior. Journal of the American Medical Association, 263 (22), 3051-56.

Diekstra, R. F. W. (1993). The epidemiology of suicide and parasuicide. Acta Psychiatrica Scandinavica, 371 (Suppl), 9-20.

Diekstra, R. F. W. (1989). Suicide and the attemptes suicide: an international perspective. Acta Psychiatrica Scandinavica, 80 (Suppl. 354), 1-24.

D'Oliveira, A. F. A. (2000-2002). Dados do núcleo de atenção ao suicídio (2000-2002). Instituto Philippe Pinel, Rio de Janeiro. Recuperado em dez 12, 2006, disponível em: http://portal.saude.gov.br/portal/arquivos/pdf/ suicidio.pdf

Dorpat, T. L., Jakson, J., \& Ripley, H. (1965). Broken homes and attempted and completed suicide. Archives of General Psychiatry, 12 (12), 213-6.

Freitas, G. U. S., Rapeli, C. B., \& Botega, N. J. (2004). Perspectiva psiquiátrica. In B. G. Werlang \& N. J. Botega (Eds.), Comportamento suicida (pp.107-121). Porto Alegre: Artmed.

Furtado, E. F. (1998). Dependência química e psiquiatria infanto-juvenil: um desafio atual. In Associação Brasileira de Psiquiatria (Org), Cidadania e direito à saúde mental (pp.147-155). São Paulo: Frôntis. 
García-Portilha, M. P. G., Martinez, P. A. S., Garcia, J. B., \& Corujo, P. G. Q. (1997). Fatores sociodemográficos. In J. B. García, J. C. G. Seijo \& P. A. S. Martinez (Eds.), Prevención de las conductas suicidas y parasuicidas (pp.46-53). Barcelona: Masson.

Greer, S. (1964). The relationship between parental loss and attempted suicide: a control study. British Journal of Psychiatry, 110, 698-705.

Hawton, K., \& Catalan, J. (1987a). The problem of attempted suicide. In Attempted suicide: a pratical guide to its nature and management (2nd ed., pp.7-25). New York: Oxford University Press.

Hawton, K., \& Catalan, J. (1987b). The circumstances surrouding attempted suicide. In Attempted suicide: a pratical guide to its nature and management (2nd ed., pp.26-49). New York: Oxford University Press.

Lester, D. (1989). Restricting methods for suicide as a means of preventing suicide: the case of drugs. Perceptual and Motor Skills, 68 (1), 273-4.

Lopes, C. S., Mari, J. J., \& Warcwald, C. L. (1991). Morbidade psiquiátrica em pacientes usuários de drogas. Revista da Associação Brasileira de Psiquiatria; Associação Psiquiátrica da América Latina (ABP-APAL), 13 (4), 161-9.

Marconde Filho, W., Mezzaroba, L., Turini, C. A., Koike, A., Motomatsu Júnior, A., Shibayama, E. E. M., et al. (2002). Tentativas de suicídio por substâncias químicas na adolescência ejuventude. Adolescencia Latinoamericana, 3 (2), 0-0.

Muza, G. M., Bettiol, H., Muccillo, G., \& Barbieri, M. A. (1997). Consumo de substâncias psicoativas por adolescentes escolares de Ribeirão Preto, SP (Brasil). I - Prevalência do consumo por sexo, idade e tipo de substância. Revista de Saúde Pública, 31 (1), 21-9.

Newson-Smith, J. G. B., \& Hirsch, S. R. (1979). Psychiatric symptoms in self: poisoning patients. Psychological Medicine, 9 (3), 493-500.

Organização Mundial de Saúde. (1979). Manual de classificação estatística internacional de doenças, lesões e causas de óbitos (9a. ed.). São Paulo: Centro da O.M.S. para a Classificação das Doenças em Português.

Organização Mundial de Saúde. (1993). Classificação de transtornos mentais e de comportamento da CID-10: descrições clínicas e diretrizes diagnósticas. Porto Alegre: Artes Médicas.
Schmidtke, A., Bille-Brahe, U., DeLeo, D., Kerkhof, A., Bjerke, T., Crepet, P., et al. (1996). Attempted suicide in Europe: rates, trends and sociodemographic characteristics of suicide attempters during the period 1989-1992. Results of the WHO/EURO Multicentre Study on Parasuicide. Acta Psychiatrica Scandinavica, 93 (5), 327-38.

Setian, N., Colli, A. S., \& Marcondes, E. (1979). Adolescência. São Paulo: Sarvier.

Teixeira, A. M. F., \& Luís, M. A. V. (1997a). Distúrbios psiquiátricos, tentativas de suicídio, lesões e envenenamento em adolescentes atendidos em uma unidade de emergência de Ribeirão Preto, São Paulo. Cadernos de Saúde Pública, 13 (3), 517-25,

Teixeira, A. M. F., \& Luís, M. A. V. (1997b). Suicídio, lesões e envenenamento em adolescentes: um estudo epidemiológico. Revista Latino-Americana de Enfermagem, 5 (Especial), 31-6.

Thatcher, W. G., Reininger, B. M., \& Drane, J. W. (2002). Using path analysis to examine adolescent suicide attempts, life satisfaction, and health risk behavior. Journal ofSchool Health, 72 (2), 71-77.

Urwin, P., \& Bibbons, J. L. (1979). Psychiatric diagnosis in self: poisoning patients. Psychological Medicine, 9 (3), 501-507.

Vansan, G. A. (1999). Aspectos epidemiológicos comparativos entre tentativas de suicídio e suicídios no município de Ribeirão Preto. Jornal Brasileiro de Psiquiatria, 48 (5), 209-15.

Vansan, G. A., \& Fávero, R. V. (1987). Comunicações diretas da intenção suicida. Revista da Associação Brasileira de Psiquiatria; Associação Psiquiátrica da América Latina (ABP-APAL), 8 (3), 97-101.

Werneck, G. L., Hasselmann, M. H., Phebo, L. B., Vieira, D. E., \& Gomes, V. L. O. (2006). Tentativas de suicídio em um hospital geral no Rio de Janeiro, Brasil. Cadernos de Saúde Pública, 22 (10), 2201-2206.

Wise, M. G. (1992). Reações de ajustamento e transtornos do impulso sem outras especificações (soe). In J. A. Talbott, R. E. Hales \& S. Yudofsky (Eds), Tratado de psiquiatria (cap. 19, pp. 446-456). Porto Alegre: Artes Médicas.

Recebido em: 6/11/2006

Versão final reapresentada em: 29/10/2007

Aprovado em: 3/12/2007 